๑) Journal club

\section{MULTIVARIATE SIGNAL INTEGRATION}

A fundamental aspect of biological systems is that they are multivariate: cells receive, integrate and respond to hundreds or thousands of concurrent environmental cues. Signalling networks provide the computational and integrative framework for cells to process and respond to these cues, and the contribution of kinases or other signalling molecules to a change of phenotype or cellular behaviour depends on the state of the signalling networks.

Indeed, kinase activation can initiate different cellular decisions depending on the pre-activation state of the network, as shown in a seminal paper by Janes et al. They showed that Jun N-terminal kinase (JNK) activation can be anti- or pro-apoptotic depending on the network state when cells receive growth factor cues. Therefore, to describe and predict a cellular response to a perturbation (such as a drug or genetic lesion) studies must be carried out in the context of the cell's multivariate network state. Because this depends on cues in the environment of the cell or tumour, the molecular roles of many genes and proteins are only valid in a specific area. In fact, a recent study by Wu et al., showing that JNK signalling can cooperate with deletion of Scribble or Ras in adjacent cells to cause cancer in Drosophila melanogaster, suggests that multivariate signal integration can even depend on multiple cells working in a coordinated manner. Thus, we must move towards the systems-level study of signalling.

Finally, as cancer and other complex diseases are diseases of networks rather than of individual molecules, we must establish network-level drug targets and diagnostic markers. Indeed, the success of an inhibitor treatment is likely to depend on the network state of tumour cells, as suggested by three recent studies on the effectiveness of BRAF kinase inhibitors (by Hatzivassiliou et al., Heidorn et al. and Poulikakos et al.), which make divergent observations that might be due to the complex multivariate and integrative nature of signalling systems and networks.

Rune Linding Cellular \& Molecular Logic Team, The Institute of Cancer Research (ICR), Section for Cell and Molecular Biology, London, SW3 6JB, UK. e-mail:linding@icr.ac.uk

The author declares no competing financial interests.

ORIGINAL RESEARCH PAPERS Janes, K. A. et al.

A systems model of signaling identifies a molecular basis set for cytokine-induced apoptosis. Science 310, 1646-1653 (2005) | Wu, M. et al. Interaction between $R_{a s}{ }^{\sqrt{12}}$ and scribbled clones induces tumour growth and invasion. Nature 463, 545-548 (2010) | Hatzivassiliou, G. et al. RAF inhibitors prime wild-type RAF to activate the MAPK pathway and enhance growth. Nature 464, 431-435 (2010) | Heidorn, S. J. et al. Kinase-dead BRAF and oncogenic RAS cooperate to drive tumor progression through CRAF. Cell 140, 209-221 (2010)| Poulikakos P. I. et al. RAF inhibitors transactivate RAF dimers and ERK signalling in cells with wild-type BRAF. Nature 464, 427-430 (2010) 